\title{
Etymologisches aus den ugrischen Sprachen
}

\author{
Wog. uxadas, urssälax̨t-
}

In wog. (Munk. VNGy IV: 2 S. 309) N uräs 'rontás; Behexung', (Tšern.) uras, uras-uj 'мифологическое существо, предвещающее несчастье, оборотень', (Kann. Wog. Volksd. V 118, 299-300) So urads 'böses Vorzeichen', LO ùràs hat man ein syrjänisches Lehnwort gesehen, dessen Original (Wichm.-Uot.) V L U ures, I ures 'u. ist z.B. ein Kind, das in sein Bett nässt, kranke Henne (V); Schimpfwort für eine unsaubere Frau (IL); durch den bösen Blick bezaubert, "verdorben» (z.P. Hund) (U); Geist, der sich in weissen Kleidern vor dem Tode eines Verwandten od. eines Freundes zeigt (I)' darstellt (s. Dávid Fokos, NyK LXII 102--105 und Károly Rédei, SLW 94-95). In Kannistos Textsammlung

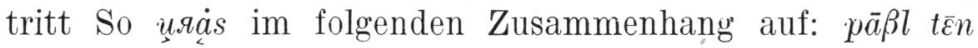

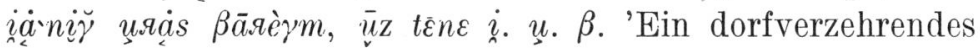
grosses böses Vorzeichen erfahre [eig. mache] ich, ein stadtverzehrendes gr. b. V. e. i.', "so dass die Leute des ganzen Dorfes, der ganzen Stadt sterben" (s. Wog. Volksd. V 118, 299-300). Es sind (a.a.O. S. 300) auch weitere die Bedeutung beleuchtende Beispiele angeführt: So när man ůås $\beta \bar{a} r \dot{e} \gamma^{\curvearrowright} \cdot n$ ? 'machst du ein ưgás?' (sagt man einem Kind, das unausgesetzt weint: $)_{\text {sagst }} \mathrm{du}$ den Tod deines Vaters oder deiner Mutter voraus?»), ńàßsam wa плачет, чего-нибудь неладно будет' (näml. den Eltern des Kindes, sie werden sterben od. dgl.); LO sampālum lös̆i,

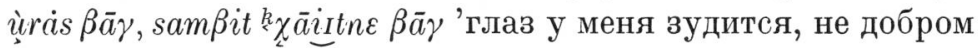
зудится, слез ворожит' (bedeutet Tränen). In einemi ähnlichen Zusammenhang tritt uräs auch in der Textsammlung Munkácsis auf: paul tēnè jäny' uräs am tēl ta värsèm usw. 'Falu 
emésztő nagy veszedelmet szereztem ím én bizonyára' (indem ich sah, dass in meine Fischreuse ein grosser Rentierochse eingegangen war usw.; s. VNGy IV 238-239). Nach dem Gewährsmann ist uräs ein muträ ural ūsna lātin 'varázslattal rontó szô' (s. VNGy IV: 2 S. 183). Da das Verb (Munk.Szil.) ūsi 'untergehen, sterben' und das Partizip 'usna also u.a. 'das Sterben' bedeutet, ]ässt sich muträ ural ūsna lätin wohl mit 'durch Zauberei, Hexerei verursachtes Sterben' übersetzen, und uräs bedeutet anscheinend auch hier genauer 'böses Vorzeichen'.

Neben diesem Substantiv kommt aber auch ein Verb vor,

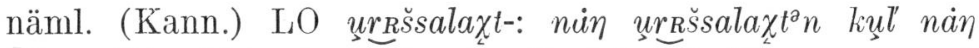
$\bar{o}^{\prime} \breve{s}_{(s n}$ 'Du bist ein spukender Teufel, du', 'Ты пугальный

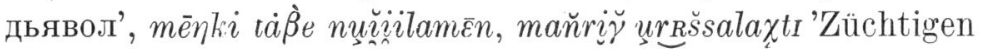
wir ihn, weil er spukt', 'Мы его колотим, почто он пугает' (s. Wog. Volksd. V 114, 296), (Mskr.) LO urssälaxt-' '»чудиться», spuken' (besonders ein mutràn 'Hexenmeister, der dem Menschen Verderben bringen kann'; die Spukenden erschrecken in der Nacht Rentiere der Menschen, und sie erscheinen oft auch den Hausleuten im Traum), So urssälaxt'spuken (ein Verstorbener, erscheint im Traum und erschreckt den Schlafenden)'. Dieses Verb ist eine reflexive Ableitung, und sein Grundwort *urssal- kann, wenn das Substantiv wirklich ein syrjänisches Lehnwort ist, von syrj. (Wichm.Uot.) S ursalni, V ursavni 'verderben', (Fokos-Fuchs) V ursavni 'einem einen Schaden anzaubern, behexen, mit dem bösen Blick schädigen', vomd'źavni-u. 'durch den bösen Blick schädigen, auf diese Weise einen Schaden anzaubern' übernommen sein.

Die syrjänische Herkunft der in Rede stehenden Wörter, die man nur aus den nördlichen Mundarten kennt, ist jedoch nicht sicher, denn auch im Ostjakischen gibt es eine Wortsippe, mit der sie zusammengehören köınen, näml. (Paas.

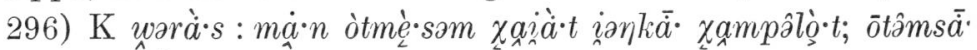
$\bar{u} i{ }^{\prime} \cdot n$ warà.s 'ich sah im Traum einen Menschen (aus dem Boot) ins Wasser fallen; im Traum sahst du etwas Schlechtes'; (Karj.-Toiv. 239) Ts. uəràs: (Folkl.) ùxèm uaràs kièř̌žà rōBâstànà 'bellst du mir den Tod an?', Kr. (Folkl.) uòtș̣̂ éna 
uaràsà ùtàn 'die Stadt möge aussterben', Ni. uị̣̂, 'Spuk; Vorzeichen, Erscheinung', Kaz. uiras 's 'Spuk', u. uદ'rț 'spuken', O ự̦à.s 'Vorzeichen?' (böser Traumı u.a.; der Gewährsmann war über das Wort nicht sicher), u. uai 'ein im Hause auftretendes, in der Wand klopfendes Tier, Wurm, sagt den Tod voraus'; DN uəràsțpad, Ni. uị̣̂asțţa, Kaz. ui̊rasıst, 'spuken'. Semantisch kommen die Wörter einander nahe, denn sie sind ja sozusagen Synonyme; man beachte besonders die Bedeutung der Dialektform von Ni. und ferner die ähnlichen Ausdrücke ostj. Kaz. uira's uع'rt̆ 'spuken'

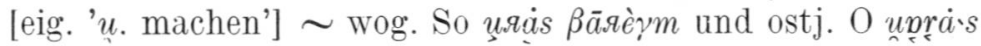
uai $\sim$ wog. (Tšern.) uras-uj [eig. 'u.-Tier']. Der Vokal der ersten Silbe der ostjakischen Wörter geht auf *ə zurück, das in Ni. und Kaz. nach $u$ durch das labialisierte $i, ?$ v vertreten ist; das $\underline{p}$ in 0 upràs ist unregelmässig (s. Karjalainen, OL 289). Steinitz schreibt in dieser Stellung $\breve{u}$, s. Ostj. Vok. 89-90. Pápay hat in ONGy $u$ geschrieben, mit dem er einen dem schwedischen $u$ ähnlichen Vokal gemeint hat (s. o.c. S. LXXX), und auch Reguly, dessen Texte bekanntlich die ausgestorbene Sygva-Mundart vertreten, hat gewöhnlich $u$, z.B. (Páp.) vur 'Blut', vural (Poss.-Suff. der 3. P. Sg.), (Reg.) url id., (Páp.) vurți, urt' 'rot', (Reg.) urti, urt id., (Páp.) vus 'Spalt': vusl, (Reg.) vussl id., usw. (s. Pápay-Bekes Wörterverzeichnis 86 und dieselben Wörter bei Karjalainen, OL 185 sowie Steinitz, Ostj. Vok. 89). Dem ostj. * entspricht ja bekanntlich wog. ${ }^{*} \breve{\imath}(>$ LO So $i$ ). Lautlich können die wogulischen und ostjakischen Wörter also nicht leicht alte etymologische Entsprechungen voneinander sein. Wog. una as lässt sich dagegen ungezwungen von ostj. uira's usw. herleiten; das ostj. $\stackrel{i}{i}, \stackrel{i}{(}(=\breve{u})$ wurde bei der Entlehnung durch wog. (Kann.) u, (Munk.) $u$ ersetzt, vor dem das anlautende $u$ (> $>$ ) schwand. Wog. *urssal-, das Grundwort von So urssälaұt- usw. könnte selbstverständlich eine wogulische Ableitung sein, aber es liesse sich auch von dem ostjakischen Verb herleiten. Der Unterschied im Vokalismus der nichtersten Silben ist durch die Synkope des Vokals der zweiten Silbe verursacht worden. Ein ähnliches Verhältnis wie bei uras urssalaxt- begegnet im folgenden Fall: (Munk.- 
Szil.) N pūmaś 'mulatság; Unterhaltung', (Kann.) KU pūmaš 'потешный, смешной; spasshaft, lustig, lächerlich, komisch', KM рйтмй, Sо рйmаs' 'весёлый; froh, fröhlich, lustig', LO pūmàs: $\bar{a} \bar{n} n^{u} m p$. '»мне диво»; es wundert mich', (Kálmán, RLW 207) So Sy pürnaš 〜 pùmáś 'lieb, froh, lustig' < ?syrj. (Fokos-Fuchs) V Ud pomet's, Le pomet's 'помочь; Hilfe, Beistand' (<Russ.) (s. Rédei, SLW 131; nach Kálmán, RLW 206-207 Wog. < ?Russ.) (Kann. Wog. Volksd. II 625,814$)$ KM pumśal- 'belustigen', (Mskr.) So pūmšal'sich liebevoll erinnern', LO pūmśălaxt- 'sich wundern', So püms̆alaxt- 'bewundern', (Bal.-Vachr.) N nумсялахтункве, J пөмщля'ӓххтәх 'увлекаться, интересоваться', (Romb. 323) пумсялахтунгкве 'увлекаться' < ? syrj. (Fokos-Fuchs) V pome (t'savni 'helfen'. Das Verb, das Rédei (a.a.O.) und Kálmán (l.c.) nicht erwähnen, könnte auch eine wogulische Ableitung sein. Bei Rombandeeva (a.a.O.) kommt auch eine Form пумасьлангкве 'увлечься' vor, deren Anfang mit dem Substantiv pümaś lautlich übereinstimmt.

Die nördlichen Wogulen und Ostjaken haben ja Jahrhunderte lang in enger Nachbarschaft gelebt, und so bilden sie, wie Kannisto bemerkt (FUF VII 187), sowohl geistig wie auch in materieller Hinsicht ein einheitliches Kulturgebiet, in dem die Nationalitätengrenze nicht viel zu bedeuten hat. ${ }^{1}$

${ }^{1}$ Kannisto zeigt in seinem Aufsatz, dass in den nordwogulischen Benennungen des Bären (Munk.) ńūrèm-uj (ńurèm-uj), ūnt-uj 'réti állat, erdei állat', 'Wiesentier, Waldtier' die ersten Komponenten ostjakische Lehnwörter sind und dass nürem-uj eigentlich 'Sumpftier' bedeutet (über die Benennungen s. jetzt näher Kann. Wog. Volksd. IV 9, 422, V 159 u. 319, 187 u. 333, 216 u. 349; das Wort für 'Wiese' hat dagegen in der ersten Silbe ein kurzes $u$ : LO ńu̧um, s. Wog. Volksd. VI 195, 328). Ausserdem stellt er fest, dass auch das als Name des Bären gebrauchte (Munk.) N ńūrmè zum 'Wiesenmann' auf einer Volksetymologie beruhen muss. Als kleine Ergänzung sei erwähnt, dass es eine solche Benennung in der lebenden Volksdichtung sicherlich nie gegeben hat. Der Ausdruck kommt in einem Bärenlied vor, das ursprünglich Reguly in der westwogulischen Sprachform aufzeichnete und das Munkácsi mit Hilfe seiner Gewährsperson in die LO-Mundart umtranskribierte. Die betreffende Stelle lautet: (Reg.) Nurmong kum kälä kuor-

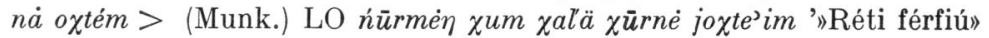
(medve) hallható zaj szélébe érkezem', 'An den Rand des Lärmes 
Es hat natürlich auch gegenseitige sprachliche Beeinflussung stattgefunden, aber besonders waren die Wogulen dabei der empfangende Teil. Dies zeigen, wie Steinitz ganz richtig bemerkt hat, auf lexikalischem Gebiet die zahlreichen ostjakischen Wörter der nordwogulischen Volksdichtung, die sogar die Gewährsmänner als ostjakisch erkannten; das Umgekehrte ist nach ihm viel seltener (s. Wog. Vok. 167). Da nun dern so ist und da wog. uadis usw. und ostj. uiras semantisch einander so nahe kommen und das erstere sich auch lautlich leicht von dem letzteren herleiten lässt - z.B. bej der Übernahme des ersteren von ostj. (Reg.) Sy. *vuras, *uras könnte ja *vu-, *u- durch u ersetzt worden sein muss man bei der Entscheidung der Frage nach dem Ursprung des wogulischen Wortes ratürlich auch das ostjakische Wort mit in Betracht ziehen. Wahrscheinlich haben die Wogulen das Wort wirklich aus dem Ostjakischen entlehnt. Falls sie dann später auch das syrjänische Wort kennenlernten, fiel es lautlich und semantisch mit dem aus dem Ostjakischen übernommenen zusammen.

\section{Wog. $\operatorname{lon} \dot{s}, \operatorname{sa} \dot{s}$}

Mit ung. les 1. 'lauern, nachstellen, nachspähen', 2. Volksspr. 'vár; warten', 3. 'Hinterhalt, Lauer, Anstand' gehört wog.

des "Wiesenmanns» (des Bären) komme ich' (s. VNGy III 334, 335). Reguly hat zu der Stelle die Erläuterung: 'slišna na pohosach chodjit' (s. VNGy III: 2 S. 291). Da in den darauffolgenden Versen eine Herde von sieben Pferden erwähnt wird, kann (Reg.) Ńurmong kum nur einen wirklichen 'Wiesenmann', den Besitzer einer Wiese bezeichnen, dessen Pferde auf der Weide umhergingen. Weil es sich aber hier um ein Bärenlied handelt, hat Munkácsis Gewährsperson in Ńurmong kum die erste Komponente der obenerwähnten Benennung des Bären vermutet, deren eigentliche Bedeutung ihr unbekannt war, und hat Ńurmong durch ńürmè (man beachte das lange $\bar{u}$ in der geschlossenen Silbe) ersetzt. Das ńürmè $\chi u m$ '"Wiesenmann" (Bär)' ist also nur eine gelegentliche, bei der Übertragung des Liedes in die LO-Mundart entstandene und offenbar auch bloss dann gebrauchte volksetymologische Bildung, die aber in der schriftlichen nordwogulischen Auffassung des Liedes weiterlebt. 


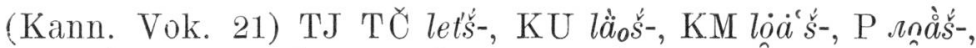
VN $ı \grave{a} s_{-}$, VS LM làšs, LU laš-, LO So lāśs- 'lauern', ?ostj. (Táp.Beke) N lāśi (Reg. lazsí, laschi) 'Anstand', lāssi- 'lauern' (s. MSzFE 402) zusammen. Es ist fraglich, ob das ostjakische Wort eine direkte Entsprechung der anderen ist, und zwar deshalb, weil es nur im Norden bekannt ist und weil also die Möglichkeit besteht, dass es ein wogulisches Lehnwort darstellt. Das Wort kann ursprünglich ein 'les; lauern' bzw. 'les; Lauer, Anstand' bedeutendes Nomen-Verbum gewesen sein (s. a.a.0.). Für diese Ansicht spricht seinerseits auch der Umstand, dass ferner im Wogulischen ebenfalls ein Substantiv von derselben alten Wurzel vorkommt, und zwar (Kann. Wog. Volksd. VI 285) VN lǖîšs, LU lonš 'скрад, Rejserhütte, in der man auf Enten lauert', LO läs etwa 'Anstand, Hinterhalt': ‘ $\chi u^{\prime} l a \bar{s}$ ' Anstand des Unterweltsfürsten' (in einem Rätsel, gemeint ist der Bog'en des Fang-

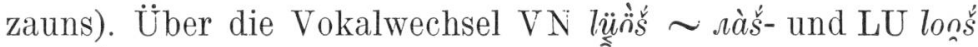
$\sim$ las-s. Steinitz, Wog. Vok. 313.

\section{Ostj. ånlatmiil}

In Pápai-Munkácsi, Déli osztják szójegyzék 16 ist ostj. ånlatmiit : Torom-čoot å. '»isteni снег видима» (látható az égi hó)', 'Himmelsschnee ist sichtbar' mit wog. (Munk.) N ānkwäti, T ånlåtål 'szemlél, tekint', 'blicken' verglichen. Iie verschiedenen Quellen enthalten offenbar keine andere Angabe über das Verb, denn im Wörterbuch von Steinitz 141 ist nur dieser einzige Beleg:*ålat-: ånlatmiil mit derr obigen Beispiel angeführt; der Vergleich mit den wogulischen Verben ist als zweifelhaft bezeichnet.

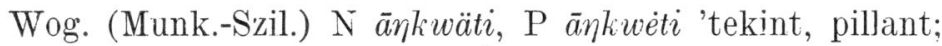

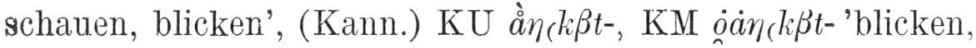
schauen' (s. JSFOu 58: 3 S. 6), (Mskr.) P à̀ $k^{2} t$-, So $\left.\bar{a} \eta_{(G} \beta a\right)^{-}$ usw. id. ist ja ein regelmässiges vordervokalisches Wort, und es hat eime genaue Entsprechung im Ostjakischen: (Karj.Toiv. 37) V ónjìrrttä́r 'blicken' usw. (s. Steinitz, Ostj. Wörterbuch 139-140). Mit diesem kann ja das obenerwähnte ostj. 
*ålat-, das anscheinend hintervokalisch und wohl ostostjakisch ist, tatsächlich nicht leicht verbunden werden, und im Wörterbuch von Steinitz wird denn auch auf das ostjakische Verb nicht hingewiesen. Das soeben erwähnte vordervokalische wogulische Wort ist aus den Tavda-Mundarten nicht bekannt, denn dort kommt nur das hintervokalische (Munk.) årlåtål

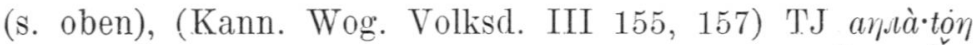

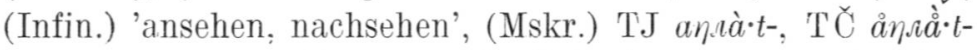
'смотреть; sehen, hinsehen, hinblicken' vor. In vereinzelten Fällen können von ein und demselben Wort zwei Varianten, eine vordervokalische und eine hintervokalische, nebeneinander vorkommen, z.B. (Kain.) KU pä $\chi l-$, KM pä $\chi l-$, LO So pókl- usw. 'bersten; aufbrechen, zu strömen anfangen',

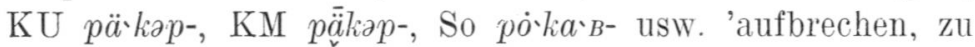
strömen anfangen', LO so pỏktul 'Riss, Spalte' (s. FUF XXX 263-264), (Mskr.) TJ T' püxmet- 'aufgehen, aufplatzen (Geschwür, Eiterbeule) (TJ); aufgehen, ausschlag'en (Blatt) (TČ)' T.J TC̆ póxmpt- 'aufgehen, aufplatzen (Geschwür, Eiterbeule) (TČ); aufgehen, ausschlagen (Blatt) (TJ)', TJ tä̈rpóxmpts : kzapp t. '»лодка проломилась»', 'das Boot barst,

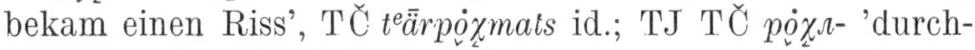
brechen (die Zähne) (TJ); aufbrechen, zu strömen anfangen (die Flüsse im Frühling) (TČ)'. Im Ostjakischen kommt nur

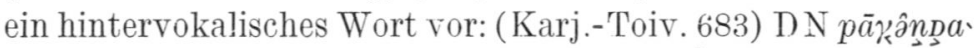
'zerspringen; aufplatzen, aufgehen', Vj. pāk k̇ânțà 'aufplatzen' usw., im Ungarischen dagegen sowohl ein hintervokalisches als ein vordervokalisches Wort, näml. fakad 1. 'ausschlagen, treiben, spriessen, sprossen', 2. 'quellen, hervorquellen, entspringen, entstehen' usw. und fekély 'Geschwïr, Abszess' (s. MSzFE 177, 191) ${ }^{1}$. Diese Wörter sind onomatopoetischen Charakters (s. MSzFE a.a.O.), das gilt aber nicht von TJ

${ }^{1}$ In (Bal.-Vachr.) N сарункве, J сӓрйәx, сӓрхәх 'тереться, ломатьса (о льде)', (Munk.) T sarlat- 'mázol; anstreichen' < syrj. *zir-: zirtni usw. 'reiben, zerreiben usw.' (s. Rédei, SLW 155) liegt keine unregelmässige Vokalvertretung solcher Art vor, dass in der Form von T ein a statt

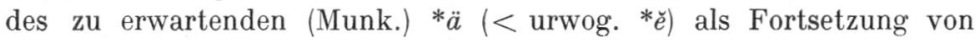
syrj. $\underset{i}{i}$ aufträte, denn T sarlat- ist ja ein tatarisches Lehnwort (s. Kannisto, FUF XVII 175). 


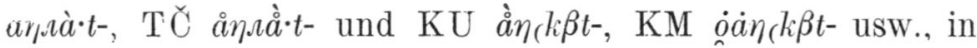
denen auch der inlautende Konsonantismus voneinander abweicht. Diese wogulischen Wörter können also nicht etymologisch zusammengehören. Dagegen erinnern wog. TJ

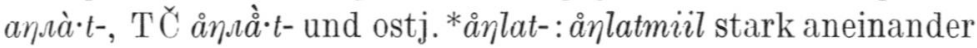
und passen auch semantisch gut zusammen, weshalb man sie nicht gerne trennen möchte.

Ung. reá, rá, rajta, róla

Ung. reá, rá 'auf ihn, darauf', rám 'auf mich' usw., -ra, -re (Endung des Sublativs); rajta 'daran, darauf, darüber; an ihm (ihr), auf ihm (ihr)', rajtunk (in der alten Literatur rohtonc) 'auf uns usw.' usw.; róla 'von ihm (ihr), über ihn (sie)', rólam 'von mir, über mich' usw., -ról, -ről (Endung des Delativs) hat man auf zwei Weisen etymologisch erklärt. Einerseits sind sie verbunden worden mit ung. rokon 'verwandt; Verwandte(r)', in der alten Literatur 'prope, non procul; propinquus, proximus; agnatus, cognatus, proximus', wog. (Munk.-Szil.) N rāwi 'közeledni; nahen', råwn'-ut 'rokon; an-

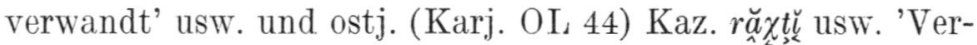
wandter', (Paas.) K rax åsm 'Verwandter seitens der Frau', J

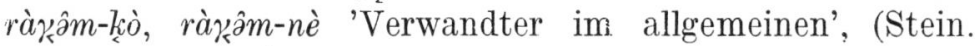

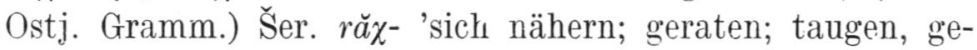
fallen; dürfen', răхtə 'Verwandter' (s. Gedeon Mészöly, MNy VI 433 ff., XXIV 308, Zoltán Gombocz, MNy VII 24, Miklós Zsirai, MNy XXIII 317, Géza Bárczi, SzófSz. 251, 252, 260, A Tihanyi apátság alapítólevele mint nyelvi emlék 31-32, 191--194, MNy LXVII 130 und Éva B.-Lőrinczy, A Königsbergi töredék és szalagjai mint nyelvi emiék 154-155 sowie die an diesen Stellen angeführte Literatur). Andererseits habe ich sie in FUF XXXI 347-353 mit dem folgenden wogulischen Worte verglichen: (Munk.-Szil.) T rån 'kivül; ausser': ämännèl-r. 'rajtam kívül; ausser mir', P rånkh̊̊: $r$. ōti 'külön lakik; allein wohnen', (NyK XXIV 21) täu jēkwät-poål rånkhè ōli 'ő külön él a feleségétől (он от

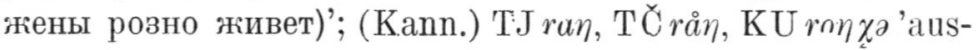




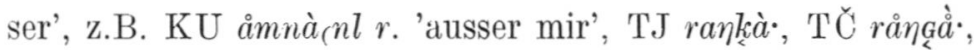

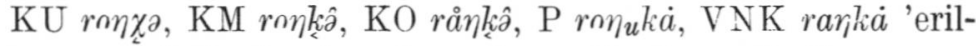
leen, zur Seite (in einen besonderen Haufen, z.B. die grossen Fische sammeln): getrennt, für sich (z.B. von seiner Frau wohnen, in den Wald gehen, Jagd treiben, etwas kaufen)', z.B. 'TJ nìtìnäl r. asànt 'er wohnt getrennt von seiner Frau' (s. auch JSFOu 58: 3 S. 23 und MSFOu 127 S. 192). Meinem Gedanken hat Péter Hajdú beigestimmt (s. Bevezetés az uráli nyelvtudományba 127), während diese Zusammenstellung nach Bárczi (MNy LXVII 130) lautlich und semantisch nicht befriedigend sei und während nach István Papp (Unkarin kielen historia 154; Tietolipas 54, i.J. 1968) die Endungen des Delativs und Sublativs aus Adverbien oder Postpositionen entstanden sind, deren gemeinsames Grundwort ein Nominalstamm roko- > ro $\gamma_{0}$ - 'Nähe, nahe' (vgl. rokon) war (s. auch Mészöly, Ómagyar szövegek nyelvtörténeti magyarázatokkal 240--241). Da nun also die Ansichten über die Etymologie der genannten Wörter und Kasusendungen auseinandergehen, dürfte es nicht unangebracht sein, auf die Frage vom Wogulischen aus zurückzukommen.

Weil von dem wogulischen Wort nur eine einzige Beugungsform vorkommt, ist es schon früh als Adverb gebraucht worden. Es hat eine deutliche lativische Bedeutung, aber auf den ersten Blick sieht es so aus, als ob es keine Endung hätte. Im Wogulischen hat die Endung des Lativs $*_{-\gamma}(<$ $*_{-}$) zwei Formen. Einerseits hat sich das $*_{-} \gamma$ dem vokalischen Stamm angeschlossen und erscheint mundartlich als $-\gamma$, $-\beta,-\ddot{z}$ oder aber ist mit dem vorangehenden Vokal zu $-i,-i$, $-\bar{a},-\bar{\varepsilon}$ usw. verschmolzen; andererseits hat sich das ${ }^{*}-\gamma$ mit einem darauffolgenden Vokal - also ${ }_{-}-\gamma_{3}$ — dem konsonantischen Stamm angeschlossen, und das $* \gamma$ ist im Nord- und Ostwogulischen bewahrt, während es in den südlichen Mundarten zu $\chi, \chi$ geworden und in den westlichen mit dem vorangehenden Vokal assimiliert worden ist. Ein gutes Beispiel bieten die folgenden Wörter, die etymologisch zusammenge-

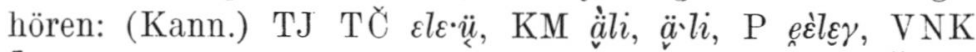
äli usw. 'nahe', KM älyam 'auf mich' und TJ $\varepsilon l \chi_{u}$, Tठ $\varepsilon l \chi$,

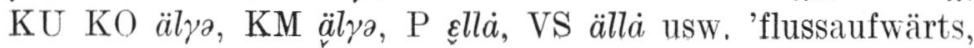


nach dem oberen Lauf des Flusses zu', TČ ellìl, KU KO älyäl' usw. id. (s. MSFOu 127 S. 177 ff., 183 ff., Wog. Volksd. II 771). Die beiden Formen der Endung kamen schon im Urwogulischen vor. Die letztere erscheint gewöhnlich bei Wörtern, bei denen der Endung noch ein Formelement folgen kann (vgl. KU KO älyäl' usw.), aber wie man aus den in MSFOu 127 S. 183 ff. angefühirten Beispielen sieht, gibt es auch Ausnahmen. Bei dem in Rede stehenden Wort sind TJ rar, TČ rån die älteren, regelmässigen Formen, denn der Schluss-

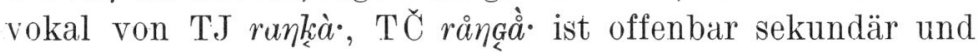
beruht auf Akzentuationsverhältnissen; ein ähnlicher sekundärer Vokal kommt auch bei einsilbigen Verben nach dem Pluralcharakter $l$ vor, z.B. (Munk.) TO mout, TU métát

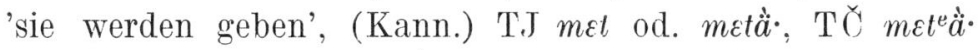
id., Bāstà 'sie sahen', îstä̀- 'sie kamen' (s. FUF XXXI 352 und Wog. Volksd. III 158). In den Lativen des 'Typs TJ TČ $\varepsilon l \varepsilon \cdot \ddot{u}$ usw. (s. oben) ist die zweite Silbe in den TavdaMundarten betont (s. die Beispiele in MSFOu 127 S. 177 ff.). Wenn sich nun in dem in Rede stehenden Worte die Endung $*_{-\gamma}$ einem vokalischen Stamm *ranks angeschlossen hätte,

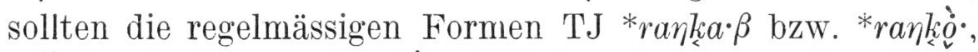
TC *ränkå $\beta$ bzW. *ränks *ranki usw. lauten. Es liesse sich nun zwar denken, dass in einem derartigen alten Adverb in $\mathrm{P}$ das $*_{-} \gamma$ geschwunden wäre und der vorangehende Vokal zu $\dot{a}$, dem gewöhnlichen Schlussvokal, geworden wäre und dass in VNK und in den Konda-Mundarten - $i,-i$ zu $-\dot{a}$ bzw. -a reduziert worden wäre. Dagegen erscheint es gar nicht glaubwürdig, dass in den Tavda-Mundarten der betonte Schluss der Formen geschwunden wäre, so dass eine Entwicklung TJ *ranka $\beta$ bzw. ${ }^{*}$ rank $\dot{o}^{\circ}>{ }^{*}$ rank $>$ ran usw. stattgefunden hätte.

Aber auch das wirkt nicht besonders glaubwürdig, dass in dem in Frage stehenden Worte an einen konsonantischen Stamm * rark- die Endungsvariante *- $\gamma_{3}$ getreten wäre, so dass eine Form *rark- ` $_{3}$ entstanden wäre. Ich habe kein Beispiel gerađe von einem solchen Fall, aber der folgende Lativ erinnert jedenfalls an die angenommene Form in der Weise, dass darin vor der Endung ${ }^{*}-\gamma_{3}$ der Palatalklusil $k_{i}$ gestanden 
hat: (Kann.) TJ TČ $l \varepsilon \chi$ 'um, rund herum, auseinander',

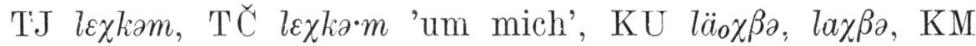

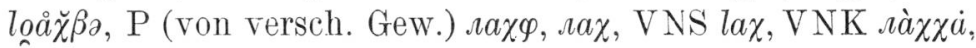
лахu $\chi \dot{a}$, VS $7 a \chi_{u} \beta \dot{a}$, LU la $\chi_{u}$ 'um, rund herum usw.', LU

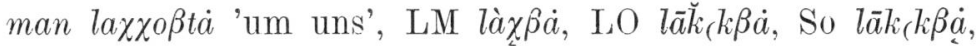

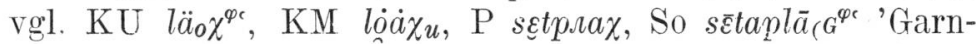
fitze' (s. MSFOu 127 S. 187, 188), (Mskr.) KM Dual lóà (kpi,

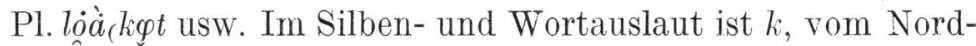
wogulischen abgesehen, allgemein zu $\chi$ geworden, und das ist auch hier geschehen; das ${ }^{*} \gamma$ der Endung ist teils mit dem vorangehenden Konsonanten assimiliert worden und in einigen Fällen mit ihm verschmolzen, teils aber hat es mit dem vorangehenden labialisierten $\chi_{u}$ ein $\chi \beta$ ergeben. Bei der Betrachtung des Adverbs TJ ran, KU ronxz usw. ist ferner zu beachten, dass von der Verbindung eines Nasals und des homorganischen Klusils, wenn sie in den Silben- und Wortauslaut geraten ist, der Nasal häufig vor allem im Süd-, Ost- und Westwogulischen geschwunden ist, und das hat besonders dort stattgefunden, wo die Verbindung ständig im Auslaut gestanden hat. Da z.B. die Ordinalzahlen als Attribut und - zwar wohl seltener - als Prädikat gebraucht wurden, trat das Sufrix $*_{-n t 3}>*_{-n t}$ immer im Wortauslaut auf, und so lautet das Suffix -t, -it, z.B. (Kann. Vok. 31, 136) TJ Tð $\varepsilon^{i} t, \mathrm{KU} \bar{a} t$ usw. 'fünf' $\sim \mathrm{TJ} \varepsilon \cdot t a t, \mathrm{KU} \bar{a}\left(t t, \mathrm{P} e_{n} \dot{\varepsilon}_{(} t t\right.$, So $a \cdot t i D$ usw. 'der fünfte'; aber im Translativ, wo das -nt- in intervokalischer Stellung auftrat, ist der Nasal bewahrt, z.B. (Kann.

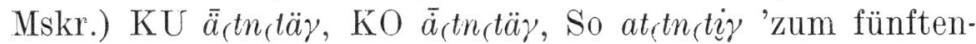

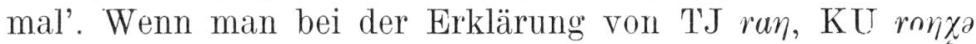
usw. von der Form *rank- $\gamma_{3}$ ausgeht, würde man also mit vollem Recht erwarten, dass der Nasal * $\eta$, weil * $\eta k$ immer nur irn Silbenauslaut auftrat, geschwunden wäre, und dass $* k$, entsprechend wie im vorhergehenden Wort TJ TČ $l \varepsilon \psi$ usw., zu $\chi, \chi$ geworden wäre. Die Entwicklung hätte also allem Anschein nach zu einer F'orm geführt, die stark von der tatsächlich vorkommenderi abweichen würde.

Die Formen TJ ran, KU ronxə usw. lassen sich dagegen ohne Schwierigkeiten so erklären, dass sich in ihnen dem konsonantischen Stamm *ran- eines alten Substantivs *rans 
die Endungsvariante ${ }^{*}-\gamma_{3}$ angeschlossen hat. Da im Wogulischen das alte inlautende ${ }^{*} \eta$ mundartlich in einigen Wörtern bewahrt ist (s. z.B. György Lakó, NyK LI 170-172, A magyar hangállomány finnugor előzményei 50-52 und Matti Liimola, FUF XXXI 110-116), kann man mit Recht annehmen, dass in dem in Rede stehenden Wort das $* \eta$ noch zur Zeit des Anschlusses der Endungsvariante ${ }^{*}-\gamma_{3}$ erhalten war. Die Endungsvariante ${ }^{*}-\gamma_{3}$ kam zuerst in den längeren Formen wie TČ clki $v^{\prime}, \mathrm{KU} \mathrm{KO} \ddot{a} l \gamma \ddot{a} l^{\prime}$ usw. (s. oben) vor, und es dauerte eine Zeit, bis sie sich auch in den kürzeren Formen

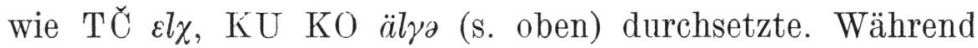
dieser Periode erschien das $* \gamma$ in ihnen teils in postvokalischer, teils in postkonsonantischer Stellung, und infolge dieses Schwankens ist die Endungsvariante ${ }^{*} \gamma_{3}$ in ein paar Wörter gekommen, in denen der Endung kein Formelement folgt (s. MSFOu 127 S. 189, 190), und zwar auch in das Wort TJ

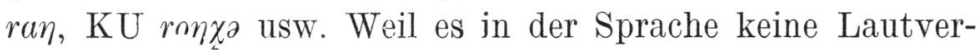
bindung $\eta \gamma$ gab, wurden die nebeneinander geratenen $* \eta$ und $*_{\gamma}$ in $*_{r a \eta}-\gamma_{3}$ durch $*_{\eta} k_{i}$ ersetzt. Es entstand so die eine feste Konsonantenverbindung enthaltende Form *ranks, die dann die anzunehmende Formvariante * raฑз verdrängte. Das *ranks hat regelmässig die heute vorkommenden Formen ergeben; in den Tavda-Mundarten schwand der Schlussvokal

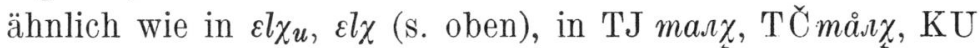
mólya usw. 'in den hinteren Teil (der Stube)', TJ tar tår 'beiseite, abseits usw.' usw. (s. MSFOu 127 S. 184, 188), und im Wortauslaut ist $\eta k$, $\eta k_{c}$ in ihnen regelrecht durch $\eta$ vertreten.

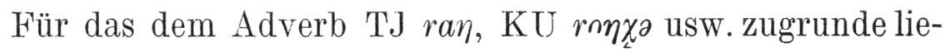
gende Wort kann man die Bedeutung 'Seite, Rand u. dgl.' annehmen. Wie ich schon in FUF XXXI 349-351 mit einigen Beispielen gezeigt habe, bedeuten manche Wörter zuweilen sogar ein und dieselbe Form eines Wortes - sowohl 'zu, auf . . zu, gegen, neben usw.' als auch 'zur Seite, abseits, seitwärts, abwärts, fort'. Aus dem Ungarischen führte ich felé 'zu, auf ... zu, gegen' und félre 'seitwärts, abseits, weg' an, die beide Formen des Wortes fél 'halb, Hälfte; Teil; Seite usw.' sind (s. jetzt MSzFE 194--196); die erstgenannte Form 
ist ein alter Lativ und bildet eine Postposition, während die letztgenannte Sublativ, also auch eine Art Lativ, ist und ein Adverb bildet. Vgl. ferner ung. (MSzFE 194) félen (alt) 'seitwärts; einsam'. Aus dem Wogulischen und Ostjakischen erwähnte ich schon (a.a.O.) Beispiele; als Ergänzung zu den Lokalkasus von wog. (Munk.) pả̄ 'Seite' usw. weise ich auf das Wörterbuch von Balandin-Vachruševa S. 87 hin, wo von demselben Wort nox 'бок' eine Menge Angaben vorliegen. Es seien aber auch einige Beispiele aus anderen Sprachen erwähnt. Fi. sivu 'Seite': Pysäköidä auto tien sivuun 'Das Auto am Rande (Illativ) des Weges (entweder innerhalb oder ausserhalb des Randes des Weges) parken', Joen molemmilla sivuilla kulki maantie 'Auf beiden Seiten des Flusses ging eine Landstrasse', Joutui sivuun selvältä tieltä 'Er geriet zur Seite von dem klaren Weg' (s. Nykysuomen sanakirja V 226); fi. syrjä 'Seite, Kante, Rand': Metsän syrjään leiriytynyt vihollisjoukko 'Feindliche Truppe, die sich am Rande (Illativ) des Waldes gelagert hatte (eig. die sich am Rande des Waldes gelagert habende feindliche Truppe)', Istusketimme autoa odotellen tien syrjässä kivellä 'Wir sassen auf einem Steine am Rande des Weges auf das Auto warteud', Kaukana syrjässä, tiettömien taipaleiden takana 'Fern abseits, hinter weglosen Strecken', Vetäytyä syrjään liike-elämästä 'Sich vom Geschäftsleben zurückziehen' (s. o.c. S. 413); tscher. (Wichm. Tscher. Texte) KB ö·rdzž usw. 'Seite': (Ödön Beke, Tscher.

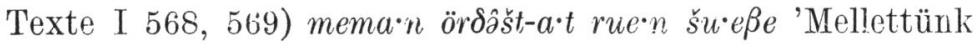
is kivágták', 'Auch in unserer Nähe (eig. neben uns) fällte man Bäume (Sing.)' [genauer: '. . . fällte man (Bäume, Sing.)':

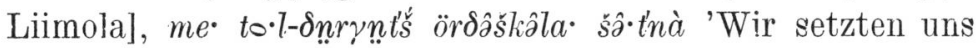
weiter abseits (vom Feuer)' (auf diese tscheremissischen Sätze hat mich Alho Alhoniemi aufmerksam gemacht); syrị. (Wichm.-Uot.) ord : o.-li usw. 'Rippe', orde usw. (Postpos.) 'zu', orded'ź usw. (Postpos.) 'bis zu, bis an' usw., aber ortse 'hinaus, heraus, fort, weg, beiseite, auf die Seite'. - Wie die Beispiele zeigen, bedeuten die lativischen Formen als Adverbien oder aber wenn sie in Verbindung mit dem Ablativ und Elativ auftreten 'seitwärts, abseits, weg' usw.', aber als Postposition (im Finnischen und in dem betreffenden tsche- 
remissischen Beispiel also in Verbindung mit dem Genitiv) dagegen 'zu, auf ... zu, neben, in die Nähe usw.', und entsprechend verhält es sich mit den anderen Lokalkasus.

Wie aus dem Obigen hervorgeht, passen wog. TJ ran, KU rnnxa usw. und ung. reá, rá, rajta, rohtonc, róla usw. wirklich sowohl lautlich wie auch semantisch gut zusammen. Das wogulische Wort lässt sich ja ausschliesslich oder, wenn man sich vorsichtiger ausdrücken will, am besten als Lativ erklären, der mit der Variante ${ }^{*}-\gamma_{3}$ der alten Endung $*_{-} \gamma\left(<*_{-}-k\right)$ schon früh im Urwogulischen von einem Substantiv * rarjs 'Seite, Rand od. dgl.' gebildet ist. Im Ungarischen wiederum ist ja $*_{\gamma}$ der regelmässige Vertreter des inlautenden fiugr. $*_{r}$. Ung. reá $\left(<{ }^{*}\right.$ rërá $<{ }^{*}$ royá $)$, rá, das ja eine alte Postposition

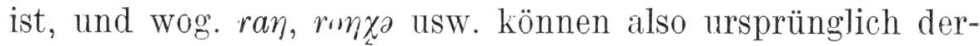
selbe Lativ desselben Substantivs sein; im Wogulischen hat sich nur eine für diese Sprache charakteristische Variante der Eridung dem Wort angeschlossen, und infolgedessen hat das lativische Adverb eine auf den ersten Blick befremdende Konsonantenverbindung erhalten. Man kann ferner mit Recht annehmen, dass das wogulische Wort, das ein Adverb ist und in Verbindung mit dem Elativ-Ablativ gebraucht wird, die Bedeutung 'zu, auf... zu, gegen od. dgl.' bekom. men hätte, falls es eine Postposition geworden wäre.

H. Paasonen hat (Beitr. 62) ling. rokon mit den obener-

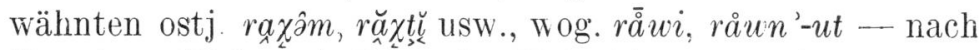

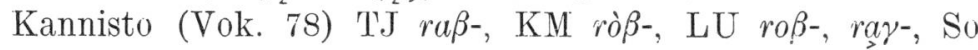
$\boldsymbol{r}^{\prime} \beta$ - 'nahe heranschleichen lassen (vom Wild)' - und sam. (Castr.) Jur. lak, rak 'близкий', rakhaj id., rakhaem 'мой родной', 'mein V'erwandter' usw. verbunden, und Toivonen (Vir. 1933 S. 306-308) und Collinder (FUV 54) haben zu diesen ferner fi. rakas 'lieb' gestellt, während Kannisto (Vir. 1933 S. 420-422) wog'. KM rå̀kâs- 'zu jemand kommen, sich jemand anschliessen (z.B. um etwas zu bitten oder um über etwas zu beraten, aber nicht z.B. böse)', råkctažt- 'einander lieben', rå. kn 'liebend' mit ung. rokon und fi. rakas zusammengestellt hat. Steinitz dagegen hat in woy. KM rå. usw. nur eine einen anderen Wechselvokal und "Wechselkonsonanten» enthaltende Nebenform von KM rò $\beta$ - (s. oben) ge- 
sehen (s. Wog. Vok. 125, 245, 311, 312), und (Fiugr. Vok. 63, Fiugr. Kons. 20) hat er das obenerwähnte ostjakische Wort: lrt. răð- usw. 'sich nähern; nahe lassen [sexuelle Beziehungen zulassen]; passen, tangen', V ră usw. 'verwandt durch die Frau [»für sexuelle Beziehungen zulässige» Angehörige der gens der Frau]; angenehm', Ni. răż usw. 'lieb' mit wog. KM råk, råkzs- $\sim$ rōw- usw., ung. rokion und fi. rakas verbunden. Wog. KM rò $\beta-\sim$ rå.k̨âswürde demgemäss also in der Vertretung des inlautenden Konsonanten einen ähnlichen Wechsel wie ung. reá $\left(<*^{*}\right.$ rëyá $<$ *royá) usw. rokon aufweisen. Es ist aber zu beachten, dass wog. KM råkำs- usw. lautlich und semantisch stark an die Form der Konda-Mundarten des ostjakischen Wortes erinnert; man vergleiche einerseits die die KU-Mundart vertretende Originalaufzeichnung (deren Transkription also nicht überprüft worden ist) (Kann. Wog. Volksd. II 460, 470)

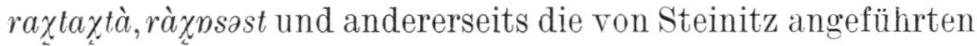
Angaben, besonders die von Irt., und ferner (Paas.) K rax̧̧િm,

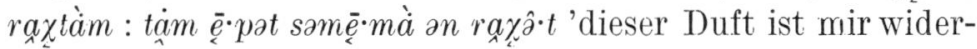
wärtig' (eig. 'dieser Duft gefällt nieinem Herzen nicht'), (Karj.-'Toiv. 800) Kam. răx td $^{\prime}$ 'sich nähern, nahe--, herankommen (konkr. und abstr.)', Kr. răx żt '»zahm», nicht scheu (Vogel, Vieh, lässt den Menschen nahe an sich kommen)'. Allem Anschein nach ist KU ràxws-, KM råkkzâs- usw. denn auch aus dem Ostjakischen entlehnt; es kann teils in der Umgangssprache, teils in der Volksdichtung übernommen sein (s. FUF XXXI 349, XXXIX 267-268). Nach SKES III 723 wiederum ist es ganz unsicher, ob die ugrischen und samojedischen Wörter mit fi. rakus etymologisch zusammengehören. Das soeben erwähnte obugrische Verb kommt freilich lautlich und semantisch der ungarischen Wortsippe reá,

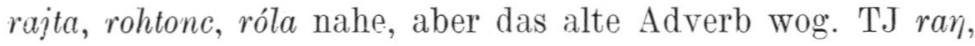

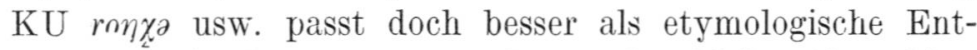
sprechung der letztgenannten. Ung. rokon bleibt mir unklar. Wenn es mit reá, rá, rajta, róla zusammengehört, ist sein $k$ erst während der Sonderentwicklung des Ungarischen entstanden. Könnte es dann nicht aus dem *y $(<* \eta)$ des ${ }^{*} r o \gamma_{3}$ entstanden sein? Bei der Betrachtung des gegenseitigen 
Verhältnisses von reá usw. und rokon wird auch auf einige andere ungarische Wörter hing'ewiesen, die einen ähnlichen oder entsprechenden inlautenden Konsonantismus zeigen (s. z.B. Bárczi, MNy LXVII 130 mit Literatur), aber auch in ihnen ist, soweit ich verstehe, der Klusil erst während der Sonderentwicklung des Ungarischen entstanden. So kann z.B. fi. räkli 'Hitze, Qalm' nicht zusammengehören mit ostj. (Karj.-Toiv. 792) Ni. rợ̆ nicht kalt)' usw., wog. (Kann. Vok. 45) TJ TČ rei, KU KM

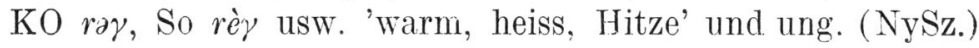
réül usw.: el-réül, el-révöl 'mente capior; in Entzückung geraten', réülés, rivülés 'mentis excessus; Entzückung', rüt, rôt, réüt usw. 'mente corripio; entzücken', (MTSz.) el-rejtözik 'elájul, elveszti az eszméletét; álhalott (»tetszhalott») lessz', d.h. 'in Ohnmacht fallen; scheintot werden' sowie rekkenő 'schwül, drückend heiss' usw. (s. SKES IV 910). Auf die Frage nach der Entstehung dieser zweifachen Vertretung gehe jch nicht ein, sondern überlasse ihre Erklärung den Spezialisten des Ungarischen. Die genannten Wörter bereiten jedoch, soviel ich einsehe, der Verbindung von ung. reá, rá, rajta, róla mit wog. TJ ran, KU ranxə usw. keine Schwierigkeiten.

Wog. näari, nä̈rèpi; ńērr

Wog. (Munk. VNGy II 677) N nä̈ri, niärèpi 'ingerel; reizen, anfreizen', (Munk.-Szil.) N ñärèi 'ingerel; hetzen', (Kann.

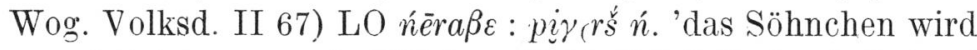
geneckt' ist von syrj. (Wichm.-Uot.) V S L P nerni, I neerni 'necken, reizen', (Gen.) PO nér- 'reizen' entlehnt (s. Rédei, SI.W 128). Als kleine Ergänzung' sei erwähnt, dass die obigen Angaben Munkácsis sowie ferner (VNGy II 677) N ńärqati 'ingerkedik', 'necken, reizen', nä̈räspi uj-êri’' 'medvebosszáének', 'Lied über die Rache des Bären' offenbar alle die LOMundart vertreten (s. VNGy I 240, 252). Aber Munkácsi hat das Wort auch aus der Sygva-Mundart aufgezeichnet: (V NGy

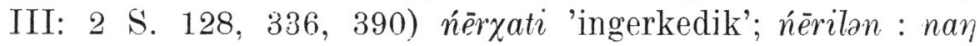


änam n'. $==$ zuitilian : 'ingerelsz', 'du hetzest mich auf' [vgl. (Munk.-Szil.) $\chi u j t i$ 'fölbujtat; aufhetzen']; Tōmil'-āyi ui

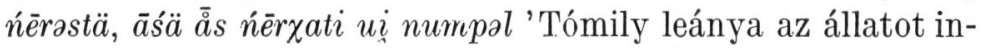
gerelte, apja is bosszantotta a medvét', 'T'omil's Tochter reizte das Tier [d.h. den Bären] auf, (und) auch ihr Vater ärgerte [Präs.] den Bären'; nérraspi : ti nérraspitä mā̄os 'wegen dieses seines Aufreizens', néeräspi uíēerì (Munk.-Szil.) néreäspi-ujèryet [o: -êrryèt] 'Lieder über die Rache des Bären'. Über das Schwanken $\bar{e} \sim \ddot{a}$ in der Bezeichnung des Vokals $\bar{e}(\bar{\varepsilon})$ s. JSFOu 72 S. 226-227. N ńêräspi, náääspi ist eine regelmässige deverbale Nominalableitung (Beispiele von dem Suffix führt Munkácsi in VNGy IV: 2 S. 174 an); néreäspi uin-êrz bedeutet also eigentlich 'Lied über das Aufreizen, Aufhetzen des Bären'. Die reflexive Ableitung ńêrxati ist hier in der intransitiven Bedeutung gebraucht und hat also kein Objekt, sondern ein die Postposition numpal 'gegen, zu, auf' enthaltendes Adverbial; das Beispiel bedeutet demgemäss eigentlich etwa '(und) auch ihr Vater verhält sich ärgernd zu dem Bären, benimmt sich ärgernd dem Bären gegenüber'.

Im Nordwogulischen kommen aber auch Adjektive vor, die

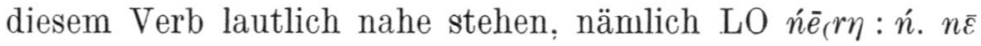
'лукавая женщина; schlaue, verschlagene, ränkevolle Frau',

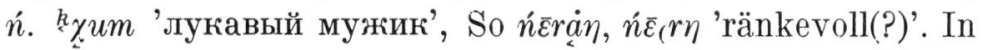
dem Adjektiv der Sosva-Mundart habe ich eine Entlehnung aus dem Ostjakischen vermutet (s. Kannisto, Wog. Volksd. V 253), während Rédei (a.a.O.) die Adjektive der beiden Mundarten mit dem oben behandelten Verb verbindet.

Das Adjektiv LO ńēern gehört anscheinend zum Wortschatz der Umgangssprache der Mundart. Kannisto zeichnete das lexikalische Material auf Wortzettel auf, und zwar, wenn es möglich war, die Formen verschiedener Mundarten desselben Wortes auf einen Zettel. Zu dem Zettel von LO ńêer $r \eta$ bekam er aber später, a!so auch während der Untersuchung

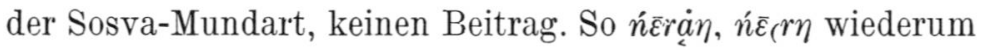
kommt nur in zwei Liedern vor: (Wog. Volksd. V 22) sāt

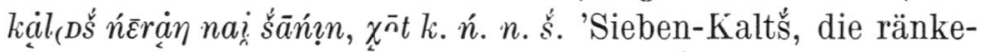
volle(?) Fürstin, deine Mutter, Sechs-Kaltš, die ränkevolle(?) 


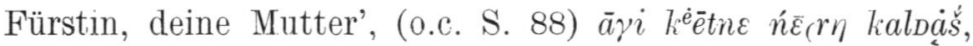
pi $\gamma$ k. n. k. 'Mädchen schickende ränkevolle(?) Kaltas̆, Jungen

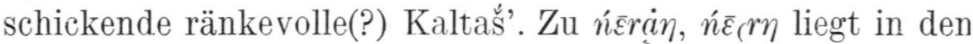
Aufzeichnungen keine Erklärung vor. An der erstgenannten Stelle ist allerdings an den Rand des Textes während der Überprüfung der Aufzeichnung mit dem Gewährsmann mit roter Tinte die Nummer 3 als Zeichen dafür geschrieben, dass sich an die Stelle eine Erläuterung schliessen sollte, aber am Ende der Textprobe findet sich keine diesbezügliche Erklärung. Dieses Adjektiv war also anscheinend den Gewährsmännern der Sosva-Mundart nicht bekannt, und obgleich es im Anschluss an LO ńér(rn mit 'ränkevoll(?)' übersetzt ist, gehört es wahrscheinlich nicht - - wenigstens nicht unmittelbar — mit diesem etymologisch zusammen. So kalDås usw. ist eine Göttin, deren Name aus dem Syrjänischen entlehnt ist (s. Rédei, SLW 103 mit Literatur). Aber auch in der nordostjakischen Volksdichtung erscheint ein Adjektiv ńéra $\eta$ als Epitheton der entsprechenden Göttin kaltoś, kattaś:ń. leattas' 'zornige kattaś', und zwar in ähnlichen Fällen

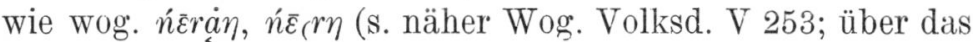
ostjakische Wort s. weiter unten). Man beachte ferner die sozusagen übereinstimmenden Ausdrücke in Gebeten an die genannten Göttinnen: (Kann. Wog. Volksd. I 271, 440) So

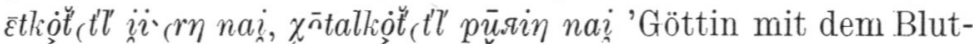
opfer der Mitternacht, Göttin mit dem Speiseopfer des Mittags' (entsprechend in LO, s. o.c. S. 280, 445) und ostj. (Pá-

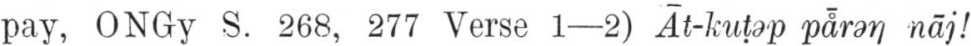

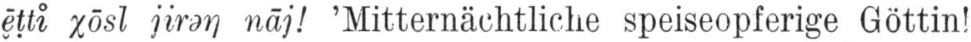
frühmorgentliche blutopferige Göttin!'. Derartige Ähnlichkeiten können nicht auf einem blossen Zufall beruhen, sondern sie erklären sich aus den nahen Berührungen zwischen

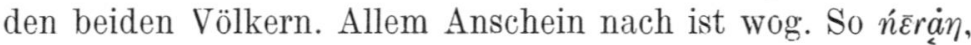
$\bar{n}_{\bar{\varepsilon}}(r \eta$, gleich wie zahlreiche andere Wörter der Folkloresprache, dann wirklich aus dem Ostjakischen übernommen und lässt sich folglich besser mit 'zornig' übersetzen.

Das Verb ńér-usw. gehört, nach der Verbreitung zu urtellen, zu der jungen Schicht der syrjänischen Lehnwörter. Wenn das Adjektiv LO ńēern mit ihm zusammengehört, ist es eine mit dem Suffix $-\eta$ gebildete wogulische Ableitung. 
Über die deverbalen Nominalableitungen dieser Art, die häufig einen lautmalenden Charakter haben, s. Magdolna Sz.Kispál, A vogul igenév mondattana 43-49. Die Zahl der in Munkácsis und Kannistos Textsammlungen vorkommenden Belege ist verhältnismässig gering, und nach der Ansicht Sz.-Kispáls war das Suffix zur Zeit des Sammelns dieser Texte schon veraltet. ${ }^{1}$ Wie zu erwarten ist, stimmt die Be-

${ }^{1}$ Sz.-Kispál führt (a.a.O. S. 46) die folgenden Fälle in einem besonderen Moment an: (Munk. VNGy III 326) N lünt-sak, vās-sak mēsin jān 'lúdbél módjára, réczebél módjára kacskaringós folyóhoz', (Kann.

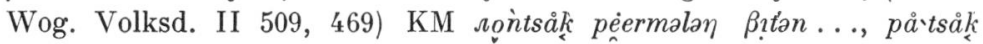

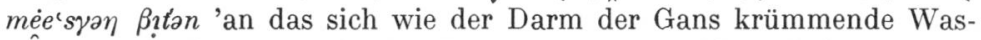
ser..., an das sich wie der Darm der Ente schlängelnde Wasser',

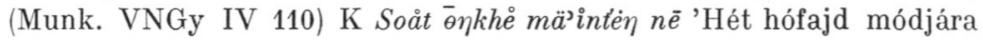
nevető nő', 'Wie sieben Schneehühner lachende Frau'. Ihrer Ansicht nach ist das grammatikalische Verhältnis des Verbalnomens und der vor ihm in der Grundform stehenden Bestimmung in diesen Fällen nicht klar. Sie hat recht, denn in den vorliegenden Beispielen handelt es sich

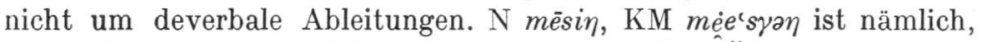
trotz der gegebenen, leider leicht irreführenden Übersetzung 'sich schlängelnd', ein possessives Adjektiv von (Munk.-Szil.) N mēsi 'Windung', (Kann. Wog. Volksd. IV 146, 473, VI 97, 255) So mési 'Biegung des Flusses'. Auch KM péermala $\eta$ ist offenbar ein denominales possessives Adjektiv. Zu Grunde liegt allerdings ein Verb peer-, und -mal ist ein zusammengesetztes Ableitungssuffix, aber es bildet hier ein Substantiv, das Grundwort der Ableitung auf -ə $\eta$; vgl. (Munk.Trócs.) N pērmil 'göngyöleg' sowie (Bal.-Vachr.) N nермил, J пермәл' '1. бинт; 2. повязка; 3. портянка', N пермилын 'обёрнутый usw.'. Auch

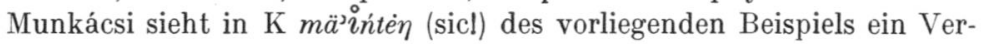

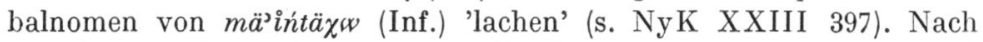

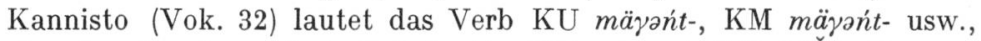
aber daneben kommt ein Substantiv KM $m \ddot{a} \gamma_{1} \Gamma^{\prime} t^{\prime}$ 'Gelächter' vor, und

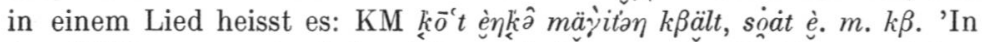
der Hütte, wo sechs Schneehühner lachen, in der Hütte, wo sieben Schneehühner lachen', eig. 'In der Hütte mit dem Gelächter von sechs Schneehühnern, ...' (s. Wog. Volksd. I 93-94, 402). Es wirkt nun glaubwïrdig, dass auch (Munk.) mä’int'è trotz seines inlautenden $\mathrm{Na}$ sals denominal ist, vgl. z.B. (Tšern.) N movint 'смех', (Bal.-Vachr.) $\mathrm{N}$ мувиньт, J мӓгт́, мӓвт́, мӓгант id. Nebenbei sei bemerkt, dass das ganz kurze, nur sechs Verse enthaltende Lied in VNGy IV 110, worin das in Rede stehende Beispiel vorkommt, kaum ein Schicksalslied ist, sondern wahrscheinlich nur ein Bruchstück des Liedes der Göttin ïßßarnai (s. Kann. Wog. Volksd. I 93-96, 401-403), wo das obenerwähnte mäjità auftritt. 
deutung der Ableitung allgemein mit derjenigen des Verbs zusammen. Ausnahmen von dieser Regel bilden (Kann.) KU

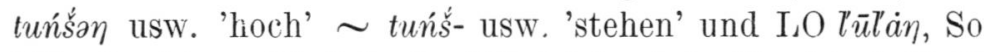

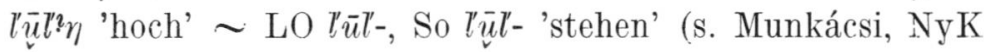
XXIV 27, D. Szabó, NyK XXXIV 421, Kannisto, Wog. Volksd. VI 258, Sz.-Kispál, o.c. S. 43), aber tuńš́ə haben sich sozusagen von den genannten Verben abgesondert und bilden schon selbständige Adjektive; neben ihnen kommen auch mit dem Äquativsuffix -it, -t gebildete Ableitungen vor, z.B. (Kann.) LO l'üriıt 'so hoch wie; Höhe', KU tu'ńs št id. (s. Wog. Volksd. II 174, VI 258). Der Bedeutungswandel 'stehend' > 'hoch' ist ausserdem einfach und durchsichtig, während ein Wande] 'neckend, reizend, aufreizend usw.' > 'schlau, verschlagen, ränkevoll' viel komplizierter wäre. Das Verb ńêr- bedeutet 'necken, reizen, aufreizen usw. (z.B. mit prahlenden, beleidigenden Worten)' (s. Munk. VNGy III: 2 S. 336). Im Satz (VNGy I 252) LO taw ānèm ness ńärèstä, āem at mistä 'он меня только подразнил; хотел мне отдать, не дал (o engem csupán ingerelt; akarta nekem adni, de nem adta)' bedeutet das Verb allerdings gewissermassen '(durch Betrügen, Täuschen) necken, reizen', und weiter unten wird man sehen, dass einer von Kannistos Gewährsmännern der P-Mundart ein anderes Verb für 'necken, reizen' wirklich mit dem Wort 'обманывать' übersetzt hat. Eine Entwicklung '(durch Betrügen, Täuschen) neckend' > 'betrügerisch' > 'schlau, verschlagen, ränkevoll' wäre also wohl an sich möglich, das würde aber voraussetzen, dass das Verb nér- irgendwo im Gebiet der LO-Mundart ausschliesslich oder wenigstens vorzugsweise gerade in der erwähnten Bedeutung gebraucht worden wäre, wodurch seine Ableitung *nérà den vermuteten Bedeutungswandel hätte durchmachen können, und dass das so entstandene Adjektiv $n \bar{e}_{c} r \eta$ 'schlau usw.' dann sich verbreitet hätte. Weil aber ńērund seine verbalen Ableitungen in allen zur Verfügung stehenden Angaben --- sogar in dem soeben angeführten Beispielsatz - mit 'дразнить; necken, reizen, aufreizen usw.' übersetzt sind und weil ńēräspi 'das Aufrejzen' bedeutet, bleibt es ganz unsicher, ob eine solche Entwicklung wirklich stattgefunden hat und ob also das Adjektiv LO ńéerr, trotz 
der lautlichen Ähnlichkeit, in der Tat mit dem Verb neêretymologisch zusammengehört.

LO ńécr $r \eta$ erinnert aber auch an das obenerwähnte ostj.

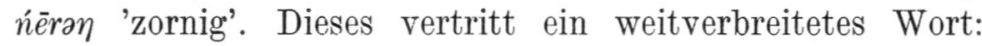
(Karj.-Toiv. 628) Ni. neer', Kaz. ńe`r', 0 ńe’r 'Mürrischkeit(?), Widerstand', V ńîrà' 'unbändig, eigenwillig, ungehorsam; bösartig, boshaft', Vj. ńīrəy' 'bösartig, boshaft (gew. vom Bären); unartig, quengelnd (Kind)', Trj. ńīrà' 'unartig, ungezogen (Kind od. Erwachsener); schlau, heuchlerisch', Kaz.

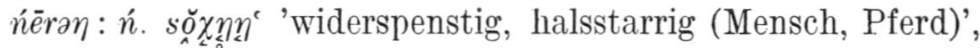

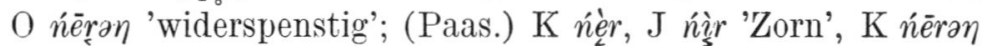
(sic!), J niịran 'zornig'. Eine Entlehnung aus dem Ostjakischen kommt schwerlich in Betracht, da LO ńe $\bar{e}_{c} r \eta$ semantisch von den nordostjakischen Adjektiven abweicht. Es könnte aber wohl die alte Entsprechung des ostjakischen Wortes sein; man beachte die Bedeutung 'schlau, heuchlerisch' in der Trj.-Mundart, die derjenigen von LO ńé(r ganz nahe kommt. Das ostjakische Wort hat in der ersten Silbe einen Vokal $e$; es gehört zu den Fällen, in denen in den östlichen Mundarten ein $i$ erscheint (über diese s. Karjalainen, OL 163 ff., 282-283, Steinitz, Ostj. Vok. 70-73, Toivonen, FUF XXXII 129132). Als Entsprechung von ostj. ${ }^{*} e$ erscheint auch sonst wog. $* \bar{\imath}(>\mathrm{LO}$ So $\bar{\varepsilon}, \bar{e}$ ), z.B. in den bekanriten Fällen (Kann. Vok. 41) TIJ TČ $n \bar{\imath}$, LO So $n \bar{\varepsilon}$ usw. 'Weib, Frau; Weibchen'

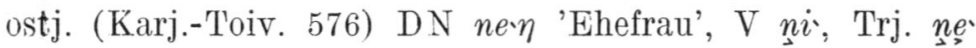

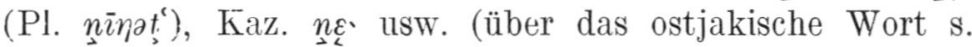
Steinitz, Ostj. Vok. 70, 108); wog. (Kann.) KU KM pīl'-, VNK $p \bar{e} l$-, LO So $p \bar{\varepsilon} l$ - usw. 'durchdringen, scharf sein (z.B. Messer)' 〜 ostj. (Karj.-Toiv. 787) DN pe ttà 'stechen; schnei-

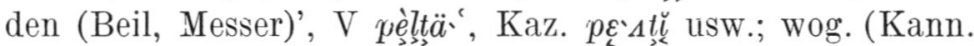

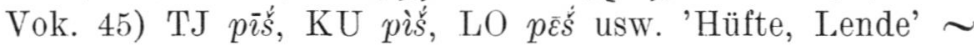
ostj. (Karj. OL 167) DN pe•t', V Vj. pirt', Ni. peș́, Kaz. pe‘ś, O piș 'Schenkel'. Die Schwäche dieser Verbindung besteht darin, dass das wogulische Adjektiv LO ńécrn wenigstens vorläufig nur aus einer einzigen Mundart aufgezeichnet ist.

Munkácsi führt aber in VNGy II 676 ein Verb ńārtaxti 'ingerkedik' an, und in V NGy I 252 heisst es in einer Erläu-

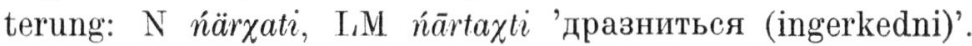


Das betreffende Lied (VNGy I 100-127) hat ursprünglich Reguly in der westwogulischen Sprachform aufgezeichnet, und in seinem Text erscheint an der in Rede stehenden Stelle

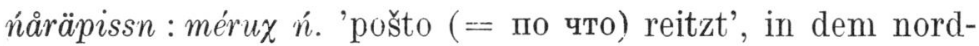
wogulischen Text Munkácsis wiederum nääépäslèn 'ingereltél' (s. V NGy I 114, 115, 241). Das in Regulys 'Text auftretende Verb und (Munk.) LM ńārtaxti gehören etymologisch zusammen, und ihre Entsprechung ist (Ahlqv.) K nurtelam 'schimpfen, höhnen'. Kannisto hat das Wort aus mehreren Mundarten aufgezeichnet: (Mskr.) KU ńärtəl- 'verhöhnen(?), verspotten(?)' (z.B. ein Reicher, der von einem Armen spricht, dass dieser nichts zu essen habe, oder ein Mensch, der, nachdem das Gericht die Forderung eines anderen abgelehnt hat, diesen spottend fragt: "Warum nahmst du denn nicht dein Land von mir zurück, obgleich du das zu tun versprachst?»),

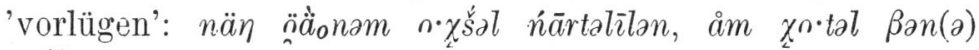
nхšà̈nam? 'ты меня деньгами налыгаешь, я откуда деньги взял? du lügst vor, dass ich Gelder habe, woher sollte ich Gelder nehmen? (eig. du lügst mich mit Geldern vor, meine woher zu nehmenden Gelder?)' (sagt ein Reicher zu einem Armen, der um Geld bittet, wenn sie in Zwietracht leben); KM ńỏärtəl- 'necken, reizen' (einen z.B. darüber, dass er vor das Gericht geriet, mit den Worten: "so musste es dir auch

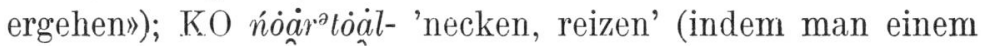

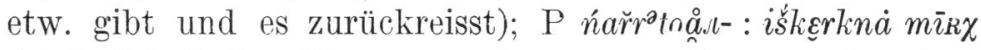

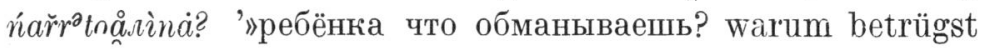
du das Kind?»' (indem du es mit einem Pfefferkuchen reizest und ihn doch nicht gibst), (nach einem and. Gew.) nartnål'»скалиться; verlachen, spotten»'; VNZ nāratüöl- 'дразнить; necken, reizen'.

Der Vokal der ersten Silbe geht auf $* \bar{a} \sim * \breve{a}$ zurück. Auffallend ist $\mathrm{KU} \bar{a}$, aber ein solches Vokalverhältnis kommt auch in einigen anderen Fällen vor (s. Kannisto, Vok. 23-24, Steinitz, Wog. Vok. 264--265). Das $t$ und $l$ sind Ableitungssuffixe, aber ihre ursprüngliche Funktion ist schwer festzustellen. Da jedoch in Regulys Beleg das $p$ ein Momentansuffix ist, bleibt als Grundwort ein Verb *nä̈r- $\sim$ *nä̈r- 'necken, reizen u. dgl.' übrig. 
Auch das so erhaltene Grundwort erinnert stark an das obenerwähnte syrj. nerni, nérni 'necken, reizen', PO nér'reizen', und man möchte es natürlich nicht gern von diesem trennen. Das letztere hat, wie die Form von PO zeigt, ein offenes $e\left(<\operatorname{ursyrj.}{ }^{*} e\right)$. Wie Rédei (SLW 46) feststellt, scheint ursyrj. ${ }^{*} e$ im Wogulischen inı allgemeinen durch $*_{\breve{e}}$, ursyrj. $*_{e}$ wiederum durch $*_{i}$ substituiert worden zu sein. Aber in einer Menge Wörter ist $*_{e}$ durch $*_{\bar{\imath}}$ ersetzt; in einem grossen Teil von ihnen folgt dem $* e$ ein $r$. Nach der Ansicht V. I. Lytkins ist in der Vorlage dieser Wörter im Frühursyrjänischen ein $*_{x}$ anzunehmen, das unter der Einwirkung des $r$ am Ende der ursyrjänischen Zeit $\mathrm{zu} * e$ wurde, und nach Rédei dürften diese Wörter schon vor dem Offenwerden $*_{x}>*_{e}$ entlehnt worden sein (s. a.a.O. S. 45--46). Wie schon oben bemerkt wurde, ist wog. nér- nach der Verbreitung zu urteilen eine junge Entlehnung, und weil urwog. * $\breve{e}$ in N zu a geworden ist, ist eine späte Übernahme auch lautlich möglich. In ein paar Fällen ist aber syrj. *e durch $* \bar{a}, * \bar{a} \sim * \breve{a}$ oder $* \breve{a}$ vertreten:

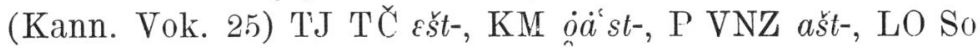
āst- usw. 'endigen (intr.) usw.' < syrj. eštini usw., PO jéšti(s. Rédei, SLW 46, 97-98); wog. (Kann.) māyr usw.: So

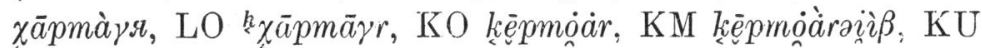

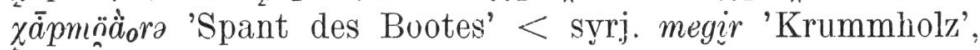
PO ńégor (vgl. Rédei a.a.O. S. 46, 122 Nr. 132 und s. FUF XXXIX 90); wog. (Kann. Vok. 133) LO iñarmak, So iāarmaG 'Seide' < syrj. (Wied.) P jermöy (s. Rédei S. 101). Betreffs des Vokals könnte also auch wog. *ńär- $\sim$ *närr- 'necken, reizen u. dgl.' aus dem Syrjänischen entlehnt sein. Es könnte jedoch wohl auch die alte etymologische Entsprechung des syrjänischen Verbs sein. Zwar kenne ich zur Zeit keinen Fall, in dem die Vokalverhältnisse ähnlich wären, aber wie auch Erkki Itkonen (FUF XXXI 309) bemerkt, gibt es nur spärlich Etymologien, die die Vorgeschichte des urpermischen $*_{e}$ erhellen.

Wog. käšlitapi, kä̋s

Munkácsi hat aus der Mundart LU ein Verb (Munk.-Szil.) käšlitapi 'odarak; hinstellen' aufgezeichnet: (NyK XXII 77) 
kit tăul šētép-tärm äkwän käšlitapi, äkwän jotlitapi 'на одну нитку две кожи вместе создевает, вместе сошьет'. Es gehört mit dem folgenden Wort zusammen: (Kann. Mskr.) TJ $k \varepsilon \check{s}$ - 'строчить (mitten auf dem Saumi von Anfang bis zu Ende eine Steppnaht nähen)', k£̧šà ·ntèm (im Sinne des Präsens) '»строчу», "торочу» (ich nähe die Tuchstücke an den Rändern zusammen)', KU kä̋̌s- 'überziehen (einen Pelz)',

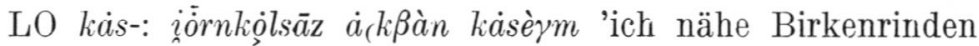
für das Rindenzelt (das "samojedische Zelt»)', sak $a^{k} i \dot{a}_{(k \beta a ̀ n}$ kàsèym '"шубу вместе шиваю" (ich nähe den Überzug und die inneren Pelzstücke des Pelzes zusammen)', So saxi kásèym id. ${ }^{1}$ Dieses Wort ist aus syrj. (Wichm.-Uot.) I kišni, U kiśnis 'anziehen (I); (den Pelz) überziehen (U)' entlehnt. In einer ähnlichen Bedeutung ist das syrjänische Wort auch ins Ostjakische übernommen (s. Toivonen, FUF XXXII 37).

Wie Rédei (SLW 113) gezeigt hat, ist auch wog. (Munk.) N kasti 'sich das Gesicht bedecken (die Frau vor den älteren männlichen Familiengliedern ihres Mannes)' aus dem genannten syrjänischen Wort entlehnt. Als kleine Ergänzung sei erwähnt, dass auch dieses Verb weiter verbreitet ist: (Kann. Wog. Volksd. VI S. 153, 295 Erk]. 103, S. 171, 309 Erkl. 10)

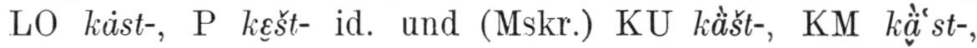
KO käst-, So käst- id. Wie a.a.O. S. 309 bemerkt ist, lebte die in Rede stehende alte Sitte zur Zeit der Reisen Kannistos nur noch bei den Nordwogulen und in einigen Gebieten an der Konda.

Matti Limola $\dagger$

${ }^{1}$ Auch (Munk.-Szil.) P käsi 'szeg; säumen', (NyK XXIV 16-17)

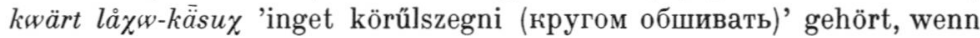
sein $s$ auf einem Schreibfehler statt $s$ beruht, hierher. 